\title{
PROBLEMÁTICA DE CONCEPTOS DE COSTOS Y CLASIFICACIÓN DE COSTOS
}

\author{
PROBLEM OF COST CONCEPTS AND CLASSIFICATION OF COSTS
}

\author{
BERnardo J. SÁNCHEz BARRAzA ${ }^{1}$ \\ Docente Asociado de la Facultad de Ciencias Contables, UNMSM
}

(Recepción: Setiembre de 2009 / Conformidad: Octubre de 2009)

\section{RESUMEN}

En la actualidad, muchas empresas e instituciones en el ámbito económico-empresarial, tienen el problema de no poder elegir una adecuada clasificación de costos, que responda a la necesidad de costeo de sus productos o servicios de forma confiable. La presente investigación pretende explicar y aclarar conceptos de costos y ejemplificarlos con casos reales de empresas, procedimientos de costeo mediante fórmulas y que el lector al final de este artículo pueda elegir el tipo de clasificación de costo que más convenga a la realidad o giro de negocio en el cual se desempeña.

Palabras claves: costo, objeto de costo, clasificación de costos.

\begin{abstract}
Actually, in economic and business many companies and institutions have the problem of choosing the appropiate classification of costs, wich let them cost theirs products and services reliably. This work wants to explain and clarify concepts of costing and gives examples about real companies and costing procedures through formulas. Also, this work should let the readers choose the sort classification of costs most advantageous to the reality or line of business in wich these work.
\end{abstract}

Key words: cost, cost object, cost classification.

Contador Público Colegiado Certificado, Director (e) EAP de Contabilidad, Docente Asociado de la Facultad de Ciencias Contables, UNMSM. E-mail: sanchez.b@pucp.edu.pe 


\section{INTRODUCCIÓN}

Es necesario que los gerentes y personal que dirigen una empresa, entiendan claramente los conceptos y clasificación de los costos, por que ello les permitirá usar adecuadamente la información contenida en reportes de costos y realizar una buena toma de decisiones gerenciales. Elegimos estudiar los conceptos y clasificación de costos por que la mayoría de empresas muchas veces no saben clasificar los costos que más le convienen a su realidad empresarial. Nuestro estudio es importante y se justifica por que una mala elección de la clasificación de costos para productos y servicios, da por resultado costos de producción unitarios errados, lo cual puede llevar a la quiebra a una empresa en el mediano plazo. Si bien es cierto hemos consultado diversos autores para esta investigación, todos tienen en común que sólo exponen la forma de clasificar los costos, pero pocos analizan la importancia de este tema, por ello pretendemos con nuestro estudio aportar un enfoque crítico y constructivo.

\section{CONCEPTOS DE COSTOS}

A continuación se definirán diversas terminologías de costos, las cuales son necesarias tenerlas presente de forma clara y precisa en las empresas.

Sistema de Contabilidad de Costos: permite mediante un conjunto de registros y cuentas contables especiales, determinar el costo de producción unitario de un objeto de costo. Cabe destacar que el sistema de contabilidad de costos no es independiente de las cuentas de la contabilidad financiera, por el contrario son relacionadas y se complementan, es decir le brinda información a la contabilidad financiera, según las necesidades que se presenten.

Mediante el sistema de contabilidad de costos se $\quad$ puede realizar el control de:

- Inventarios, el cual esta conformado por materias primas directas, productos en proceso, productos terminados, etc. El control de inventarios al igual que en la contabilidad financiera se puede lograr por medio de documentos de control como requisición de materiales, informe de recepción, informe de producción, kardex, etc.

- Activo de planta, en este rubro tenemos maquinarias, en las cuales realizamos su control por medio de la depreciación, que refleja el desgaste por uso y obsolescencia tecnológica de los activos fijos que posee una empresa.

- Montos gastados en actividades funcionales, se refiere a los fondos gastados en las áreas funcionales de producción, administración y ventas, por lo tanto teniendo una relación de los costos y gastos de estas áreas se puede realizar un seguimiento y comparación periodo a periodo y ver su evolución, y así determinaremos si aumentaron o disminuyeron, y se analizarán las causas de estas fluctuaciones.

Contabilidad de Costos: es aquella contabilidad que se ocupa de la clasificación, acumulación, control y asignación de costos a un «objeto de costo». La clasificación de costos dependerá de la realidad productiva de la empresa, actividades y procesos, por ejemplo para una empresa industrial la clasificación mas adecuada es la de costos de materia prima directa, mano de obra directa y costos indirectos de fabricación. En cambio para un servicio contable la clasificación mas apropiada será la de costos directos y costos indirectos. Cuando hablamos de acumulación nos referimos a centralizar los costos por cuentas, procesos o trabajos. El control lo realizamos de la comparación que puede efectuarse periodo a periodo. La asignación plantea el uso de un determinado método de costos que permitirá el cálculo adecuado de los costos y repartirlos a los productos o servicios.

- Objeto de costo: se define como cualquier bien tangible o intangible, susceptible de ser costeado o tener valor. Ejemplo: galleta, avión, carrera universitaria de derecho, marca (franquicia) de pizza hut, construcción de un edificio, asesoría contable, etc.

Propósitos de los Costos:

- Permite proporcionar informes referentes a costos para medir la Utilidad, la cual se determina por medio del Estado de Resultados, y Valuar el inventario, lo cual se aprecia en el Balance general.

- Brinda información para el control administrativo de las actividades y operaciones de toda la empresa, para tal fin se efectúan los Informes de Control.

- Proporciona información para la Planeación y la Toma de decisiones, que permitirá visionar al futuro con una tendencia hacia el crecimiento económico de la empresa. 


\section{FLUJO DE OPERACIONES EN DIVERSOS TIPOS DE EMPRESAS:}

\section{EMPRESAS COMERCIALES}

Compra mercaderías a a

Tipos de inventarios: Mercaderías y suministros diversos

\section{EMPRESAS INDUSTRIALES}

Compra y consume materias primas $\longrightarrow$ transform $\longrightarrow$ agrega gananci $\longrightarrow$ vende Tipos de inventarios: Materias primas, Productos en procesos, Productos terminados, subproductos, desechos, desperdicios, suministros diversos, etc.

\section{EMPRESAS DE SERVICIOS}

Presta el servicio $\longrightarrow$ agrega ganancia $\longrightarrow$ vende

Tipos de inventarios: suministros diversos como útiles de oficina y otros

\section{CONCEPTOS DE COSTO, GASTO Y PÉRDIDA}

COSTO: se define como el valor sacrificado de unidades monetarias para adquirir bienes o servicios con el fin de obtener beneficios presentes o futuros. Por ejemplo si compramos leche y luego consumimos una parte en la fabricación de helados, estamos empleando materias primas para fabricar otro bien, el cual luego de la venta nos dará los beneficios económicos.

GASTO: este concepto plantea que el gasto es un costo que nos dio un beneficio y que ha expirado, esto es desde el punto de vista que nos brindo frutos. Por lo tanto los gastos nos permiten obtener ingresos, es decir, los beneficios.

Entonces bajo este enfoque debemos pensar: ¿el costo de artículos vendidos, es decir el «costo de ventas», es un gasto por que permite la generación de los ingresos?, la respuesta es «si», por que el costo de ventas fue un costo productivo sacrificado para obtener el producto terminado que se ha fabricado. Sin embargo, no lo llamamos «gasto de ventas» sino «costo de ventas», para evitar la confusión con los gastos de ventas que sirven para impulsar el producto hacia la venta. De todo lo expuesto concluimos que, en contabilidad la palabra gasto se asocia a los departamentos administrativos y de ventas, dando por resultado los términos «gastos administrativos» $\mathrm{y}$ «gastos de ventas» (como un acuerdo contable) los cuales contribuyen indirectamente a la generación de ingresos en el Estado de Resultados y la palabra «costo» se usará generalmente dentro de los costos de producción de los bienes fabricados. Finalmente, debemos indicar que la palabra gasto también sirve en el ámbito financiero, de ahí se desprende el término "gasto financiero». Ejemplos: gastos de contabilidad son considerados gastos administrativos; gastos de comisiones a los vendedores son gastos de ventas; gastos de intereses por préstamos son gastos financieros.

PÉRDIDA: es cuando los bienes y activos que posee una empresa pierden su valor sin haber brindado ningún beneficio económico. También se refiere a que las participaciones que tiene la empresa no han producido valores compensatorios en relación a lo invertido. Por ejemplo, si a un stock de harina de trigo le salen hongos, esta materia prima ya no se puede usar en la fabricación de panetones, por lo tanto, el valor económico de la harina se convirtió en cero. Otro caso es cuando ocurre un desastre natural en alguna zona industrial, las instalaciones derruidas o afectadas pierden su valor económico, incluso aún si estuvieran aseguradas, por que las instalaciones ya están destruidas y el dinero de la aseguradora reemplazará y construirá un nuevo local.

Costo-Gasto-Pérdida

- Tenemos que destacar que el «costo» va al Estado de ganancias y pérdidas en forma paulatina a medida que se van vendiendo los productos terminados, en cambio el «gasto» va la Estado de ganancias y pérdidas de forma inmediata en el momento que se devengan los gastos periodo a periodo. Finalmente las pérdidas muchas veces se consideran como algo excepcional o poco usual y se reflejan inmediatamente de conocerse en el estado de ganancias y pérdidas, en cumplimiento del principio contable de prudencia. 
- Ejemplo: Realizamos la producción de 3000 unidades de un producto comestible, el cual tiene un «costo de producción unitario» de $\mathrm{S} / 5$, por lo tanto el costo de producción total sería: 3000unids.x S/.5 = S/.15,000.

Si 2000 unids. son vendidas, el «costo de ventas» será 2000unids.x S/.5 = S/.10,000 el cual va al Estado de Ganancias y Pérdidas. El resto de 1000unids quedan en inventarios en el activo del Balance General a un «costo» de 1000unids.x S/5 $=\mathrm{S} / .5000$. Si estas unidades no se logran vender y se malogran (por que es un producto perecible), el costo de $\mathrm{S} / .5,000$ se convierte en «Pérdida». Por otro lado si fueron necesarios algunos costos de comercialización para que los productos sean más vendibles, estos costos serán definidos como «gastos de ventas».

Finalmente, es obvio que el costo de ventas del producto es diferente al valor de venta de los mismos, por que lo segundo involucra una ganancia inmersa, por ello sería conveniente analizar: Si cada producto comestible lo vendo a S/.8 tendríamos una ganancia unitaria de S/.3 sobre el costo unitario de $\mathrm{S} / .5$, en cambio si lo vendemos a $\mathrm{S} / 4$ se estaría produciendo una pérdida de S/.1, esto es obvio por que conocemos el costo de producción unitario de $\mathrm{S} / .5$, de ahí la importancia de saber siempre el costo de producción por que éste nos permite determinar las ganancias o pérdidas que pueden generar los productos.

\section{ELEMENTOS DEL COSTO DE PRODUCCIÓN}

El costo de producción en una empresa industrial esta conformado por:

Materias primas directas (MPD), son los costos consumidos de materiales directos, los cuales son de vital importancia para la fabricación de un producto terminado. Estos costos representan un monto económico significativo respecto al costo del producto terminado. Por ejemplo, para la elaboración de un néctar de durazno en botella, la materia prima directa es la pulpa de durazno.

Mano de obra directa (MOD), son las remuneraciones consumidas y devengadas en un periodo expresadas en dinero, que reciben los trabajadores que fabrican el producto ya sea con sus manos o con herramientas. Cabe destacar que el trabajador emplea su esfuerzo físico y/o mental para elaborar el producto. Los beneficios sociales de trabajadores directos como: essalud, seguro complementario de trabajo de riesgo, gratificaciones, compensación por tiempo de servicios, etcétera, también son parte de la MOD. Ejemplo, si fabricamos camisas los costureros que unen las piezas de tela, serán mano de obra directa respecto a estos productos.

Costos indirectos de fabricación (CIF), se refiere a todos los costos que son consumidos en planta pero que su asociación respecto a los productos terminados es «indirecta», debido a que son usados en muchos productos o líneas de productos. Ejemplo, la energía eléctrica en planta es común a la fabricación de diversos productos en un laboratorio de medicinas, sin embargo es difícil asociar estos costos a aspirinas, jarabes para tos, etcétera, para ello de realiza un reparto mediante tasas de asignación empleando el concepto de prorrateo (dividir y asignar).

Fórmula de Costo de Producción del Periodo (CP):

$\mathrm{CP}=\mathrm{MPD}+\mathrm{MOD}+\mathrm{CIF}$

De la suma de los consumos de los 3 elementos obtenemos el costo de producción total, en este caso se considera que todo lo producido es terminado y no existen inventarios iniciales o finales de producción en proceso (producción no terminada).

Ejemplo, se elaboran bizcochos con MPD $=\mathrm{S} / .3000$, $\mathrm{MOD}=\mathrm{S} / .2000$ y $\mathrm{CIF}=\mathrm{S} / .7000$, el Costo de producción es $\mathrm{CP}=3000+2000+7000=\mathrm{S} / .12000$. Si se obtienen 24000 unidades de bizcochos el costo de producción unitario es S/.12000/24000unids= S/. 0.50

Fórmula de Costo de Producción de Productos Terminados en el Periodo (CPpt):

$\mathrm{CPpt}=$ Inv.Inic.Prod.Proceso $+\mathrm{CP}$ - Inv.Final.Prod. Proceso

Esta fórmula involucra la fórmula anterior incluida (CP), a la cual se le adicionan los Inventarios Iniciales de Productos en Proceso y a su vez se le restan los Inventarios Finales de Productos en Proceso.

Por ejemplo, continuando con la elaboración de bizcochos, podemos tener en un periodo «masa de bizcochos» que no ha sido horneada, por lo tanto a esta masa se le denomina producto en proceso.

Si $\mathrm{CP}=\mathrm{S} / .12000$, pero ahora tenemos Inv.Inic.Prod.Proceso $=\mathrm{S} / .1500$ y el Inv.Final.Prod. Proceso $=\mathrm{S} / .593$ entonces el CPpt será: $\mathrm{CPpt}=1500+12000-593=\mathrm{S} / .12907$

El costo de producción de los productos terminados es S/. 12907, si la producción de estos productos del periodo son $\mathrm{CP}=24000$ unids y los Inv.Inic.Prod.Proceso $=3300$ unids y el Inv.Final. Prod.Proceso $=1200$ unidades, los productos efectivamente terminados son CPpt (unidades)= $3300+24000-1200=26100$ productos terminados. Luego el Costo de producción unitario de CPPt $=$ S/.12907 / 26100unids. $=$ S/.0.494521 
Fórmula de Costo de Ventas (CV):

$\mathrm{CV}=$ Inv.Inic.Prod.Terminados $+\mathrm{CPpt}-$ Inv.Final. Prod.Terminados

Esta fórmula involucra la fórmula anterior incluida (CPpt), a la cual se le adicionan los Inventarios Iniciales de Productos en Terminados y a su vez se le restan los Inventarios Finales de Productos Terminados.

Por ejemplo, podemos tener bizcochos fabricados este periodo y también bizcochos fabricados el periodo anterior pero que no fueron vendidos, estos últimos en el presente periodo son Inventarios Iniciales de productos terminados. Finalmente, durante este periodo podemos tener bizcochos no vendidos los cuales representan los Inventarios Finales de productos terminados.

Si CPpt $=$ S/.12907, pero ahora tenemos

Inv.Inic.Prod.Terminados $=\mathrm{S} / .960$ y el Inv.Final. Prod.Terminados $=\mathrm{S} / .715$ entonces el CV será: $\mathrm{CV}=960+12907-715=\mathrm{S} / .13152$

El costo de producción de artículos vendidos (costo de ventas) es S/.13152, ahora si la producción de los productos terminados en el periodo son $\mathrm{CPpt}=26100$ unids, los Inv.Inic.Prod.Terminados= 2000 unids y el Inv.Final.Prod.Terminados $=1450$ unidades, los productos efectivamente vendidos son CV (unidades) $=2000+26100-1450=26650$ productos vendidos.

Hay que destacar que los Inv Inic.Prod. Terminados $=2000$ unids más los productos terminados $\mathrm{CPpt}=26100$ unids dan por resultado 28100 unidades disponibles para la venta, entonces si en el Inv.Final de Prod.Terminados quedan 1450 unidades (productos no vendidos que permanecen en almacén), por diferencia el resto de unidades fueron vendidas, es decir 26650 productos terminados vendidos.

* Nota: para efectos de demostrar las fórmulas no se consideran los grados de avances de los productos.

\section{CLASIFICACIÓN DE COSTOS}

Existen diversas formas de clasificar los costos, nosotros tomaremos como base la clasificación planteada por Ralph Polimeni en su libro «Contabilidad de Costos», por que la consideramos bastante completa, sin embargo nuestras clasificaciones planteadas, han sido enriquecidas con otros conceptos y experiencias en el campo empresarial de los costos.

1) Costos relacionados con los Elementos de un producto

2) Costos relacionados con la Producción

3) Costos relacionados con el Volumen
4) Costos relacionados a la Capacidad de asociarlos

5) Costos en base al departamento donde se incurrieron

6) Costos en base a las áreas funcionales y/o actividades realizadas

7) Costos en base al periodo en que los costos se enfrentan a los Ingresos

8) Costos en relación con la Planeación, Control y Toma de decisiones.

\section{1) Costos relacionados con los Elementos de} un producto

Este tipo de clasificación plantea que los elementos del costo de un producto fabricado son tres: Materia prima directa, Mano de obra directa y Costos indirectos de fabricación. Si bien es cierto ya se habían brindado los conceptos de estos elementos, ahora los definiremos de forma un poco más amplia.

Materias primas: son materiales o recursos que se emplean en producción para transformarlos en productos terminados, para ello se necesita de los costos de mano de obra directa y costos indirectos de fabricación. También se dice que son los costos de todos los materiales que con el tiempo y dentro de un proceso productivo se convierten en parte del «objeto de costo». Existen dos tipos de materias primas:

Materia prima Directa, es aquella que puede identificarse y asociarse en la fabricación de un producto, su participación es importante, principal y tiene un costo significativo en proporción al producto. Ejemplo: Tela para confeccionar un pantalón.

Materia prima Indirecta es aquella que puede ser difícil de identificarse en la fabricación de un producto, su participación es secundaria pero necesaria y tiene un costo poco significativo en proporción al producto. Ejemplo: Hilos para confeccionar un pantalón.

* Otro concepto importante es el de Materia Bruta, la cual se define como los bienes que nos brinda la naturaleza, pero en su estado natural. Por ejemplo, un árbol en su estado natural es una materia bruta, pero cuando se le tala y se le da forma de tablas se convierte en materia prima.

- La distinción entre materia prima directa e indirecta muchas veces no es fácil o definitiva, todo depende del criterio profesional de la persona que realiza la clasificación para el costeo. Retomando el ejemplo de los hilos para confeccionar un pantalón, se consideró indirecto, por el poco monto económico y/o físico que se pueda emplear de hilo en la prenda, sin embargo alguna persona de forma aguda podría afirmar que sin el hilo el pantalón no adquiere su forma 
definitiva y por lo tanto es directo, por ello tendríamos que explicarle nuestro enfoque planteado.

- En el campo del arte culinario, los chefs y bartender afirman que para que un platillo o cocktail quede con un sabor de calidad se necesitan de todos los ingredientes, por tal motivo todo material lo consideran directo.

- De lo expuesto podemos concluir, que cada empresa puede tener una forma diferente de ver y clasificar sus costos, incluso hasta en empresas del mismo giro. Además para una empresa una materia prima podrá ser directa y para otra en otro proceso productivo diferente podrá ser la misma materia prima una materia prima indirecta.

Mano de obra: es el costo económico retribuido a un trabajador por el esfuerzo físico y/o mental empleado en la fabricación de un producto. Existen dos tipos de mano de obra:

Mano de obra Directa, aquella directamente relacionada en la elaboración de un producto terminado debido a que hace el producto y puede identificarse fácilmente en la fabricación del mismo. Su participación es importante, principal y su costo puede ser significativo, dependiendo que tan intensiva sea la mano de obra en relación al producto. Ejemplo: zapatero en la confección de un calzado hecho a mano.

Mano de obra Indirecta, relacionada indirectamente en la fabricación de un producto ya que no hace el producto. Su participación es secundaria pero necesaria, generalmente participa en labores de apoyo productivo y supervisión. El costo de mano de obra indirecta será significativo si la empresa usa maquinaría con tecnología de punta, la cual necesitará personal de supervisón para estas máquinas. Por el contrario, si no existen muchas máquinas y el trabajo es más artesanal, se necesitará de poco personal de apoyo. Ejemplo: personal que ayuda trasladar los materiales, supervisores de planta, etcétera.

- Al igual que en materia prima la distinción entre mano de obra directa e indirecta muchas veces no es fácil o definitiva. Todo depende del criterio profesional de la persona que realiza la clasificación para el costeo. Por ejemplo, muchas empresas consideran que hoy casi no existe mano de obra directa, por que como muchas máquinas son automáticas y accionadas por computadoras, se afirma que poco hacen los trabajadores de forma directa. Sin embargo, otras empresas afirman que a pesar que un trabajador accione una máquina automática, ésta no se maneja por si sola, por ello el trabajador que la programa o realiza una formulación programada también será mano de obra directa. Mas aún el ingeniero químico de planta, es necesario para la formulación perfecta de una medicina, sin esta fórmula no se pude realizar la producción, bajo este enfoque su participación es directa, a pesar que él no fabrica la medicina.

- Podemos concluir que cada empresa puede tener una forma diferente de ver y clasificar sus costos de mano de obra. Cabe destacar que los beneficios sociales de los trabajadores directos e indirectos también son parte de la mano de obra según su procedencia respectivamente.

Costos Indirectos de fabricación: son todos los costos de planta que no se pueden identificar específicamente con el producto. Dentro de estos costos tenemos la siguiente sub-clasificación: Materia prima indirecta, Mano de obra indirecta, Otros costos indirectos. Ejemplos: energía eléctrica de planta, depreciaciones de planta, agua de planta, impuesto de planta, mantenimiento de planta, limpieza en planta, alquiler de planta, seguro de planta, herramientas consumibles en planta, etcétera. Los costos indirectos de fabricación pueden ser fijos, variables o mixtos, esta clasificación se explicará mas adelante.

\section{2) Costos relacionados con la Producción}

Esta clasificación está relacionada con los elementos del costo de un producto y con los principales objetivos de la planeación y el control. Se afirma que no es muy adecuada esta clasificación para la acumulación de costos por si misma, a menos que se use en concordancia con otros elementos del costo. Las dos categorías, con base en su relación con la producción son:

Costos primos: integrado por las materias primas directas y la mano de obra directa. Estos costos se relacionan en forma directa con la producción. Ejemplo: si la $M P D=S / 3500 y$ $M O D=S / 2500$ entonces el costo primo es:

Costo Primo $=$ Costo de MPD + Costo de MOD

Costo Primo $=3500+2500=6000$

Costos de conversión: son los relacionados con la fabricación o transformación de las materias primas directas en productos terminados. Los costos de conversión son la unión de la mano de obra directa y los costos indirectos de fabricación Ejemplo: si la $M O D=S / 2500$ y $C I F=S / 4000$ entonces el costo de conversión es:

Costo de Conversión $=$ Costo de $M O D+$ Costo de CIF

Costo de Conversión $=2500+4000=6500$

El siguiente esquema nos ilustra un enfoque con el cual se debe tener cuidado al usar los costos relacionados a la producción, sobre todo para evitar el doble costeo de alguno de los elementos si es que usara para fines de acumulación de costos. 


\section{COSTOS PRIMOS}

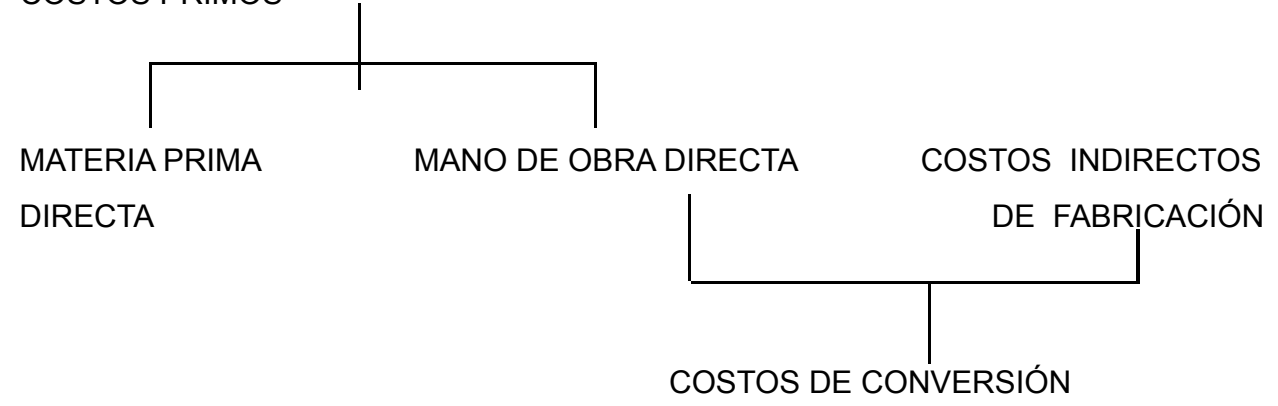

En el esquema anterior, la mano de obra directa se incluye en ambas categorías. Esto no genera una doble contabilización porque esta clasificación se utiliza para la planeación y el control, no para la acumulación. Por lo tanto, ¿qué pasaría si lo usáramos para un costeo?, por ejemplo: $\mathrm{MPD}=\mathrm{S} / 1500$, $\mathrm{MOD}=\mathrm{S} / 500, \mathrm{CIF}=\mathrm{S} / 2000$, y planteáramos: Costos primos + Costos de conversión $=$ Costo de producción, reemplazando

$\mathrm{MPD}+\mathrm{MOD}+\mathrm{MOD}+\mathrm{CIF}=1500+500+500+$ $2000=4500$, pero el Costo de producción es $\mathrm{MPD}+\mathrm{MOD}+\mathrm{CIF}=1500+500+2000=4000$, observamos que existen en el primer caso un exceso de S/500 por la doble contabilización del costo de mano de obra, lo cual es erróneo.

En cambio si tuviéramos lo siguiente:

Costos Primos + CIF ó MPD + Costos de conversión, en ambos casos obtenemos el costo de producción de S/4000, lo cual si es correcto.

\section{3) Costos relacionados con el Volumen}

Estos costos varían de acuerdo con los cambios (aumentos o disminuciones) en el volumen de producción, estos se enmarcan en casi todos los aspectos del costeo de un producto, y considera el rango relevante de producción algo importante para su explicación. Estos costos se clasifican en:

Costos variables: aquellos en los que el costo total cambia en proporción directa a los cambios en el volumen, o producción, dentro del rango relevante, en tanto que el costo unitario permanece constante. Ejemplo: si necesitamos tela por S 5 para hacer un polo (camiseta), ¿cuánto costo variable se emplearía para hacer 20 polos?

Producción de polos: 20, Costo unitario variable S/5.

20 polos $\times S / 5=S / 100$ de Costo variable total.

Si la producción baja a 12 polos, entonces 12polos $x$ $S / 5=S / 60$, observamos que el costos variable total disminuyó en $S / 40$, en cambio el costo unitario permanece constante en $S / 5$ a nivel unitario.
El costo variable total aumenta o disminuye directamente proporcional a los aumentos y disminuciones del volumen de producción.

Costos fijos: Son aquellos en los que el costo fijo total permanece constante, inalterable o no cambia en función de la producción, este hecho se cumple dentro de un rango relevante de producción (capacidad productiva de planta), mientras el costo fijo por unidad varía con la producción. Fuera del rango relevante de producción, variarán los costos fijos totales, por lo tanto ante aumentos de niveles productivos en el tiempo, todos los costos serán variables incluso los costos fijos, por que si necesita una mayor producción, el tamaño de la planta actual ya no será suficiente, entonces se tendrá que alquilar otra planta de mayor dimensión que tendrá un alquiler mayor que la planta productiva original.

Costos mixtos: aquellos costos que tienen una parte de costos fjosy otra de costos variables, a lo largo de varios rangos relevantes de producción. Existen dos tipos de costos mixtos: costos semivariables y costos escalonados.

- Costos mixtos semivariables: conformado por una parte de costo fijo que usualmente representa un cargo minimo fijo al hacer determinado producto o servicio disponible. La parte variable es el costo cargado por usar realmente el servicio.

Ejemplo. El costo de Energía eléctrica tiene un costo fijo por el derecho al servicio y una parte variable de acuerdo a los kw/hora consumidos.

- Costos mixtos escalonados: son costos fijos que cambia abruptamente a diferentes niveles de actividad productiva.

Por ejemplo, si la empresa Hyundai tiene un supervisor de planta por cada 5 automóviles diarios que produce, al cual se le remunera US\$4000 mensuales, este costo será fijo dentro de la supervisión de 0 a 5 autómoviles, si la empresa aumenta la producción a 7 automóviles el costo del supervisor no será suficiente, por que él no podrá supervisar el exceso de 2 automóviles, por ello se necesitará contratar a otro supervisor para las 2 unidades adicionales, el cual podrá ganar US\$2000, ahora en el nuevo rango relevante de producción de 0 a 7 automóviles los costos de supervisión se han 
incrementado a US\$6000, pero en este nuevo rango relevante nuevamente se convirtieron en fijos.

\section{4) Costos relacionados a la Capacidad de asociarlos}

Estos costos dependen de la capacidad de la gerencia para asociarlos con los productos, departamentos, órdenes, etc.

Costos Directos: son los costos que la gerencia es capaz de asociar con un producto o área especifica. Los costos de materia prima directa y mano de obra directa son costos directos al producto. Ejemplo: costo de cuero para un portafolio (MPD), costo de panadero para hacer pan (MOD).

Costos Indirectos: son costos que no son directamente asociables a un producto o área por que son costos comunes a muchos productos. Ejemplo: el costo de gas para el horno de una pastelería se tiene que repartir entre muchos productos horneados, se pueden repartir en base a las horas de gas empleadas en el horneado, es decir hora-máquina.

\section{5) Costos en base al departamento donde se incurrieron}

La clasificación de estos costos plantea que el costeo por departamentos ayuda a la gerencia a controlar los costos indirectos por cada departamento y a medir el ingreso. En las empresas industriales tenemos los siguientes tipos de departamentos:

DEPARTAMENTOS DE PRODUCCIÓN:

Estos departamentos contribuyen directamente a la fabricación de un producto terminado, por lo tanto son departamentos donde se dan los procesos de conversión o de elaboración. Incluye operaciones manuales realizadas por la mano de obra directa y mecánicas con ayuda de maquinaria industrial para realización del producto.

Ejemplo: tenemos Departamentos de Mezcla de materias primas, Departamento de Horneado de Masas, etc.

\section{DEPARTAMENTOS DE SERVICIOS:}

Aquellos departamentos que no están directamente relacionados con la elaboración de un producto terminado. Su función generalmente consiste en suministrar servicios a otros departamentos como los departamentos productivos y en ocasiones otros departamentos de servicios. Los costos de estos departamentos por lo general se asignan a los departamentos de producción.

Ejemplo: Departamento de Limpieza, Departamento de Mantenimiento de Maquinaria, etc.

\section{6) Costos en base a las áreas funcionales $y / o$} actividades realizadas

Estos costos son clasificados por función y se acumulan según la actividad realizada. Los costos de una empresa industrial pueden dividirse en costos de manufactura, mercadeo, administrativos y financieros. Definidos de la siguiente manera:

Costos de Manufactura o de Producción: son los costos que se relacionan con la producción de un artículo. Los costos de manufactura son la suma de las materias primas directas, mano de obra directa y de los costos indirectos de fabricación.

Ejemplo: Costos de manzanas para fabricar una jalea de manzanas.

Costos (gastos) de Mercadeo o de Venta: Aquellos que se incurren en la promoción, impulso y venta de un producto o servicio. Contablemente son llamados Gastos de ventas.

Ejemplo: Publicidad televisiva, publicidad en revistas, comisiones a vendedores.

Costos (gastos) Administrativos o de Gestión: son los costos que se consumen en la dirección, administración, gestión, control y operación de la empresa. Contablemente son llamados Gastos de administración.

Ejemplo: Sueldo de gerente general, sueldos de personal administrativo, etc.

Costos (gastos) Financieros: Estos costos se relacionan con la obtención de fondos para la operación de la empresa, o con algún costo que cobran las instituciones financieras por cuentas bancarias que tenga una empresa. Contablemente son llamados Gastos Financieros.

Ejemplo: Costos de los intereses que la compañia debe pagar por los préstamos, gastos de mantenimiento de cuentas bancarias, costos por créditos bancarios, etc.

* Nota: tenemos que destacar que esta clasificación emplea la palabra «costo», que como sabemos involucra un valor sacrificado para obtención de beneficios. Sin embargo en la profesión contable a todos estos tipos de costos se les llama "gastos», como gasto de ventas, gastos administrativos, etcétera, entonces debemos ser conscientes que todo valor sacrificado en una empresa es un costo, por que se realizó con la esperanza de obtener un beneficio presente o futuro, por lo tanto todo «costo de operatividad»(gastos operativos) de la empresa, en algún momento fue un «costo», que rápidamente se consumió y se convirtió en "gasto», por lo tanto esta palabra es la que comúnmente se emplea para definir dicho término desde el punto de vista administrativo y de ventas. Finalmente, entonces todo gasto (costo) administrativo y de ventas se incurrió para dar beneficios de gestionar la empresa de forma adecuada.

\section{7) Costos en base al periodo en que los costos se enfrentan a los Ingresos}

Aquí tenemos algunos costos que se registran o contabilizan primero como activos (Costos de capital) y luego se deducen (se cargan como un gasto) a medida que se consumen o expiran, ejemplo de estos costos serán la compra de una maquinaria y su posterior depreciación. 
Otros costos se registran inicialmente como gastos (Gastos de operación), ejemplo gastos de mantenimiento.

Esta clasificación de los costos en categorías con respecto a los periodos que benefician, ayuda a la gerencia en la medición y evaluación del ingreso, en la preparación y análisis de estados financieros y en la asociación de los gastos con los ingresos en el periodo apropiado. Las categorías son:

Costos del producto: aquellos costos que se identifican o relacionan directa e indirectamente con el producto fabricado, estos son los costos consumidos de materiales directos, mano de obra directa y los costos indirectos de fabricación. Estos costos no brindan ningún beneficio hasta que se venden los productos $y$, por consiguiente, se inventarian hasta la terminación de producto, es decir «los costos se llevan a inventarios» en los almacenes. Luego cuando se venden los productos, sus costos totales se registran como un gasto, brindando el beneficio del ingreso. Finalmente este costo es denominado costo de los bienes vendidos o costo de ventas.

Costos del periodo: son costos que no se están directa ni indirectamente relacionados con el producto, no son inventariados. Los costos del periodo se cancelan inmediatamente, puesto que no puede determinarse ninguna relación entre costo e ingreso. Hay que destacar que algunos expertos en costos afirman que indirectamente los costos del periodo si contribuyen a la generación de ingresos, por que las empresas necesitan de ellos para dirigir la empresa y lograr ventas. Estos costos están conformados por los gastos operativos de administración, gastos de ventas y ocasionalmente los gastos financieros.

8) Costos en relación con la Planeación, Control y Toma de decisiones

Estos costos ayudan a la gerencia y a los administradores en las funciones de planeación, control y toma de decisiones. Entre estos costos se pueden destacar:

\section{Costos estándares y costos presupuestados}

Los costos estándares son aquellos que deberian consumirse en un proceso de producción en condiciones normales de eficiencia productiva, es decir trabajando con calidad. El costo estándar se define como el «costo que debe ser).

El costeo estándar se relaciona con los costos unitarios de los materiales directos, la mano de obra directa y los costos indirectos de fabricación, y cumplen el mismo objetivo de un presupuesto pero a nivel unitario, en cambio el presupuesto cumple un objetivo a nivel de producción total.

Los costos presupuestados muestran la actividad «pronosticada a futuro» sobre una base de costo total y producción total en cambio el costo estándar sobre una base de costo unitario.
Ejemplo: el costo estándar por un pastel de vainilla es $\mathrm{S} / 8.00$, si necesitamos realizar un presupuesto para 15 pasteles que se piensan fabricar, los costos presupuestados serían $\mathrm{S} / .8 .00 \times 15=\mathrm{S} / .120 .00$

La gerencia utiliza los costos estándares y los costos presupuestados para planear el desempeño futuro y tomar decisiones, también para controlar el desempeño real de la empresa mediante el análisis de variaciones, es decir, la diferencia entre los costos proyectados y los costos reales.

\section{Costos controlables y no controlables}

Los costos controlables son aquellos que pueden estar directamente influenciados por los gerentes de unidad en determinado periodo. Los costos no controlables son aquellos que no se administran, o no son influenciados en forma directa por un determinado nivel de autoridad gerencial.

Ejemplo: el sueldo de un trabajador es un costo controlable, en cambio, el costo de impuesto predial de planta es un costo no controlable.

\section{Costos fijos comprometidos y costos fijos discrecionales}

Un costo fijo comprometido surge, por necesidad cuando se cuenta con una estructura organizacional básica(similar a la capacidad de planta), es un fenómeno a largo plazo que por lo general no puede ajustarse en forma descendente sin que se afecte adversamente la capacidad de la organización para operar, incluso a un nivel mínimo de capacidad productiva. Ejemplo: alquiler de planta y el costo de depreciación de equipo de planta son costos fijos comprometidos.

Un costo fijo discrecional surge de las decisiones anuales de asignación de costos para reparaciones y mantenimiento, costos de publicidad, capacitación de los ejecutivos, etc.

Es un fenómeno a término de corto plazo, que por lo general, puede ajustarse de manera descendente, y permite que la organización opere a cualquier nivel deseado de capacidad producida, teniendo en cuenta los costos fjos autorizados.

Ejemplo: el costo de publicidad televisiva depende que cuantas veces queremos que se emita un spot publicitario en un canal de televisión.

\section{Costos irrelevantes y relevantes.}

Los costos relevantes son costos futuros esperados que difieren entre cursos alternativos de acción y pueden descartarse si se cambia o elimina alguna actividad económica.

Ejemplo: el costo de materia prima para fabricar un nuevo helado de chocolate es un costo relevante, ya que se dará por la producción del nuevo producto. Los costos irrelevantes son aquellos que no se afectan por las acciones de la gerencia, los costos hundidos son un ejemplo de los costos irrelevantes. Estos son costos pasados que ahora son irrevocables, como la depreciación de la maquinaria. 
Ejemplo: los costos de licencias municipales son costos irrelevantes, si se quiere fabricar un nuevo producto dentro de las mismas instalaciones de la planta

\section{Costos diferenciales}

Es la diferenta entre los costos de cursos alternativos de acción sobre una base de elemento por elemento. Si el costo aumenta de una alternativa a otra, se denomina costo incremental; si el costo disminuye de una alternativa a otra, se denomina costo decremental. Con frecuencia los costos incrementales y variables son los mismos.

Ejemplo: el costo de materia prima directa para hacer un pantalón es $\mathrm{S} / 9.00$, cada vez que se necesite producir un pantalón más, se requiere de $\mathrm{S} / .9 .00$ adicionales o diferenciales, en este caso será un costo diferencial incremental.

\section{Costos de oportunidad}

Cuando se toma una decisión para empeñarse en determinada alternativa, se abandona los beneficios de otras opciones. Los beneficios perdidos al descartar la signiente mejor alternativa son los costos de oportunidad de la acción escogida. Cabe destacar que estos costos no se contabilizan, si no, sólo sirven para comparaciones en procesos de toma de decisiones, generalmente en proyectos de inversión.

Ejemplo: el costo de que no alquilemos nuestra planta productiva a un tercero, será el costo de oportunidad de seguir fabricando, en vez de alquilar la planta y recibir dinero por ella.

\section{Costos de cierre de planta}

Son los costos fijos en que se incurriría aun sino bubiera producción. En un negocio estacional, la gerencia a menudo se enfrenta a decisiones de si suspender las operaciones o continuar operando durante «la temporada muerta».

Ejemplo: costos de impuestos municipales y alquileres de planta, se dan de todas formas aún si no se tiene producción

\section{CONCLUSIONES}

- Luego de haber revisado los diversos conceptos y clasificaciones de costos, podemos afirmar que no existe una sola forma de clasificar los costos que responda a los requerimientos de costeo de toda empresa.

- La clasificación de costos que debe elegir una compañía depende del giro de negocio y del objeto de costo (bienes tangibles e intangibles) que brinde.

- Estudiar y comprender los diversos términos de costos permite a los usuarios de la información de costos a tomar mejores decisiones de inversión.
- Realizar una adecuada clasificación de costos permite la obtención de costos de producción reales, confiables y fidedignos.

- El uso adecuado de las fórmulas: CP, CPpt y $\mathrm{CV}$, permiten hallar los costos de producción y costos de ventas de forma confiable.

\section{REFERENCIAS BIBLIOGRÁFICAS}

1. Polimeni.R. (2000). Contabilidad de Costos. Tercera Edición. Mc Graw Hill Interamericana S.A., Colombia.

2. Horngren.C. (2007). Contabilidad de Costos. Doceava Edición. Prentice Hall Hispanoamericana S.A. México

3. Torres. G. (2006). Tratado Contabilidad de Costos por Sectores Económicos. Primera Edición. Consultores S.A. Perú.

4. Santa Cruz.A. (2008). Tratado de Contabilidad de Costos. Primera Edición. Tomo II. Instituto Pacífico. Perú. 